\title{
DEVELOPMENT OF A FINGERPRINT-BASED ATTENDANCE NOTIFICATION SYSTEM USING SIMPLE MAIL TRANSFER PROTOCOL
}

\author{
ADEJUMOBI O.K., *ADEDOYIN M. A., ADENOWO A. A., \\ SHOEWU O.O., YUSSUFF A.I.O. \\ Lagos State University, Nigeria, Department of Electronic and Computer Engineering \\ *e-mail: mary.adedoyin@lasu.edu.ng
}

Received: $8^{\text {th }}$ December 2020

Accepted $6^{\text {th }}$ January 2021

Published:24 $4^{\text {th }}$ March 2021

https://doi.org/10.47545/etrj.2021.6.1.076

\begin{abstract}
The concept of attendance monitoring has since evolved to become an integral part of every functional society. Today, in most educational institutions, students' attendance is being taken manually by lecturers on paper-based attendance registers. However, this method is time-consuming, inaccurate and may not be available for analysis when needed because the collected data has not been stored in any database. Hence, in this paper, a fingerprintbased, wireless students' attendance system using C\# and MYSQL is developed to address the problem of truancy, human error in taking attendance and impersonation. Also, a fingerprint device with the Graphical User Interface (GUI) is modelled using C\#, which was used for both enrolment and verification while MYSQL was used to model the database. The Simple Mail Transfer Protocol (SMTP) is then used to send the PDF version of the attendance records to relevant stakeholders to ascertain the students' levels of attendance. The developed system is simple, secure and cost-effective to implement. The developed system worked efficiently when experimented.
\end{abstract}

Keywords: Attendance System, C\#, Database, Enrolment, MYSQL, Verification.

\section{INTRODUCTION}

Research has shown that there is a significant relationship between students' academic performances and levels of attendance. For instance, the authors in [1] indicate in their paper the existence of some empirical evidence showing that attendance does have a positive impact on every student's performance and progression. In educational institutions, attendance has become a requirement for students to sit for examinations. The attendance management system is one of the most advanced applications in biometric technology- It cannot be forged easily because the biometric technology is secured. Biometrics is the automatic identification of a person based on his or her physiological characteristics. It includes fingerprint, facial, retinal, laughter, voice and iris [1]-[3]. Fingerprint-based biometric technology helps in eradicating the problem of impersonation since fingerprint recognition and matching is one of the simplest ways of verifying a person's identity. It requires imaging using a fingerprint sensor and comparison of the print pattern which includes the ridges and minutia points. These patterns are unique to every individual. The developed system uses the technique of fingerprint verification to automate the attendance of students. It has been proved over the years that the fingerprints of every person are unique [1]. Fingerprint authentication is accurate, safe and economical.

C\# is an object-oriented programming language designed by Microsoft that runs on .Net Framework. C\# programming language helps to develop different types of robust and secured applications [4]. MYSQL is an open-source Relational Database Management System (RDMS) based on Structured Query Language (SQL) [5]. The Simple Mail Transfer Protocol (SMTP) is an internet standard communication protocol for electronic mail transmission. Message transfer agents and mail servers usually use SMTP to send and receive mail messages. SMTP is a connection-oriented, text-based protocol in which a mail sender communicates with a mail receiver by issuing command strings and supplying necessary data over a reliable ordered data stream channel such as a Transmission Control Protocol (TCP) [6], [7].

The importance of an effective attendance management system has driven substantial research. Researchers had Adejumobi et al, 2021 
proposed and designed different methods to take attendance, manage and analyze them. For instance, NewmanFord et al. [1] assessed the impact of an electronic attendance monitoring system on academic attainment. The latter attempts to corroborate the findings of earlier work, but undertaken on a larger scale. The results of the study, an evaluation of 22 first-year modules on four different award programmes using an electronic attendance system, showed a strong correlation between attendance and academic performance. On the other hand, Salami [2] designed a fingerprint-based attendance monitoring system. The system uses Andrino Mega 2560 microcontroller board, Python's Tkinter library for its graphical user interface (GUI), and structured query language (SQL) for its database. Communication between the system modules was established using the Bluetooth technology. System test was undertaken and the outcome shows students' fingerprints were successfully enrolled and subsequently verified. However, the extent of the claimed success was not mentioned.

Notwithstanding the aforementioned, Kommey et al. [3] presents a Near Field Communication (NFC)-based system with fingerprint authentication. The proposed system, named SwyftTapp, is made of three (3) modules that includes: an API that handles communication between the other two modules, a reader for capturing fingerprints, and a web-based platform to access the system. Its implementation/testing was restricted to a department within the latter's university, hence generalization of its performance on a large scale can not be ascertained. Also, Rahman et al. The authors in [7] proposed an attendance system that marks attendance after student's biometric identification. This work uses a Minutiae algorithm to develop the identification system and implemented in the C\# programming language. According to the latter, the system was also tested on a class of students with their fingerprints matched to the database. The test thus indicates a significant result was achieved. In [6], the server is PHP modelled while MySQL and PHP are used as back-end. HTML, CSS and JavaScript are used as front-end tools. The design provides a faster and cheaper online students' attendance. The authors in [8], designed a system that is using Model, View, and Controller (MVC) architecture, and implemented it using the power of Laravel Framework. The database was modelled using MySQL. Insertions, deletions, and changes of data in the system can be done via the GUI.

In [9], a microcontroller and the LabView platform have been used to design an attendance system. The authors came up with a Real-Time Operating System (RTOS) approach to simplify the problems associated with fingerprint devices in extracting salient features and matching them. The attendance system is fast, consumes low power, and efficient. However, it has limited functionality and can only be used where the databases are small. The work in [10] combines Radio Frequency Identifiction (RFID) and Global System for Mobile Communications (GSM) technology with biometrics. Students' identification cards (ID), which are tagged with the RFID tag. The RFID tag is then matched with the database and attendance is finalized after the fingerprints are verified. The GSM module is used for sending SMS to parents regarding students' levels of attendance. The system is fast and secure but expensive and its software design is difficult. In [11], [12], Students have to swipe the RFID card along with their fingerprints to mark the attendance. The system can be accessed through the developed android application. The system is secure, has more functionality, and can be accessed remotely. However, it is costly, and the android software design is complex. The database is designed using the SQLitet tool. In addition to fingerprint biometric, vein recognition is used. The authors in [13] designed a system that allows for real-time attendance monitoring and processing using a website. Attendance records can be obtained from the website with vein recognition. This design is secure but expensive because of the complex software design involved. In [14], [15], the authors used a GSM module to send the attendance reports of every subject along with start time to the stakeholders such as parents and the head of department (HoD). This system is portable, easy to use and consumes less power but costly and has a low data rate. In [16], an attendance management system using biometrics to take attendance electronically was designed. However, the aspect of sending the PDF version of the attendance records to relevant stakeholders to ascertain the students' levels of attendance has not been investigated. Hence, different from the existing related work, in this paper, a fingerprint-based attendance notification system using a simple mail transfer protocol is developed.

The remaining part of this work is organized as follows: Section 2 presents the methodology adopted in this work. In section 3, detailed information about the obtained results has been provided. Section 4 concludes this paper.

\section{METHODOLOGY}

This work is based on the development of a fingerprint attendance notification system using SMTP. It is a fingerprint-based, wireless students' attendance system using C\# and MYSQL to address the problem of truancy, 
human error in taking attendance and impersonation. The proposed system can be used to monitor students' attendance and identify registered students. The system requires that each student enrol his/her fingerprint for the system to identify and verify if he or she is a registered student. Also, a fingerprint device with the Graphical User Interface (GUI) is modelled using C\#, which was used for both enrolment and verification while MYSQL was used to model the database. The SMTP is then used to send the PDF version of the attendance records to relevant stakeholders to ascertain the students' levels of attendance. The system is divided into two (2) parts namely; Enrolment and Fingerprint Recognition (Verification). The GUI of both parts was modelled and structured using the $\mathrm{C} \#$ programming language while the database server was modelled using MySQL.

\subsection{Enrolment}

Enrolment is the process of collecting students' information into the system's database for recognition and verification to be done.

Enrolment Procedure: Figure 1 shows the enrolment procedure as follows:

1. Start Enrolment from the Main Menu (see Figure 2) and select Reader (see Figure 3).

2. Capture Finger using the selected Reader.

3. Enrol 1 by creating the template of the $1^{\text {st }}$ Image

4. Remove your finger and press it again on the Reader.

5. Capture Finger.

6. Enrol 2 by creating the template of the $2^{\text {nd }}$ Image.

7. Remove the finger and press it again on the Reader.

8. Capture Finger.

9. Enrol 3 by creating the template of the $3^{\text {rd }}$ image

10. Capture Finger

11. Enrol 4 by creating a template of the $4^{\text {th }}$ image and merging all 3templates.

Figure 2 shows the main menu of the proposed system which involves the reader selection, verification, enrolment, GUI, capture, identification, streaming and identification interface. Figure 3 displays the reader selection interface.

The enrolment interface shown in Figure 4 is used to accept the students' information (e.g. full names, date of birth etc.) with their pictures and fingerprints that will be stored in the system's database for attendance and verification purposes. The fingerprint reader (DigitalPersona - 01279CB5) was used in this model comprises three (3) sensors namely; the Finger Line Path, the Genotype, and the Finger Capture. Hence, comparing/capturing and verifying through these sensors enhance its efficiency. A student's finger captured four (4) times were merged (see experimental examples in Figures 4-7); if the four (4) scanned/captured images are merged as one then, the capturing is successful and the details of the particular student will be saved into the database (see Figure 7) else, if there is any sign of change in that specific finger which is currently been captured once or twice, the capturing will continue till the seventh capturing process before it breaks.

\subsection{Fingerprint Recognition (Verification).}

Once the fingerprint is captured, the next step is the recognition or verification process as shown in Figure 8, which is one-to-one matching. The Verification Interface is modelled using the C\# Programming language while the database is modelled using MYSQL. The process of fingerprint verification is shown in Figure 8. Shown in Figure 9, the verification interface, which has four (4) features; to verify and mark attendance for a specific student, to convert the details and data in the attendance database to PDF and to send the details and real-time records of the attendance to a specific mail using the SMTP feature. Figure 10 shows the verification interface after valuation. 


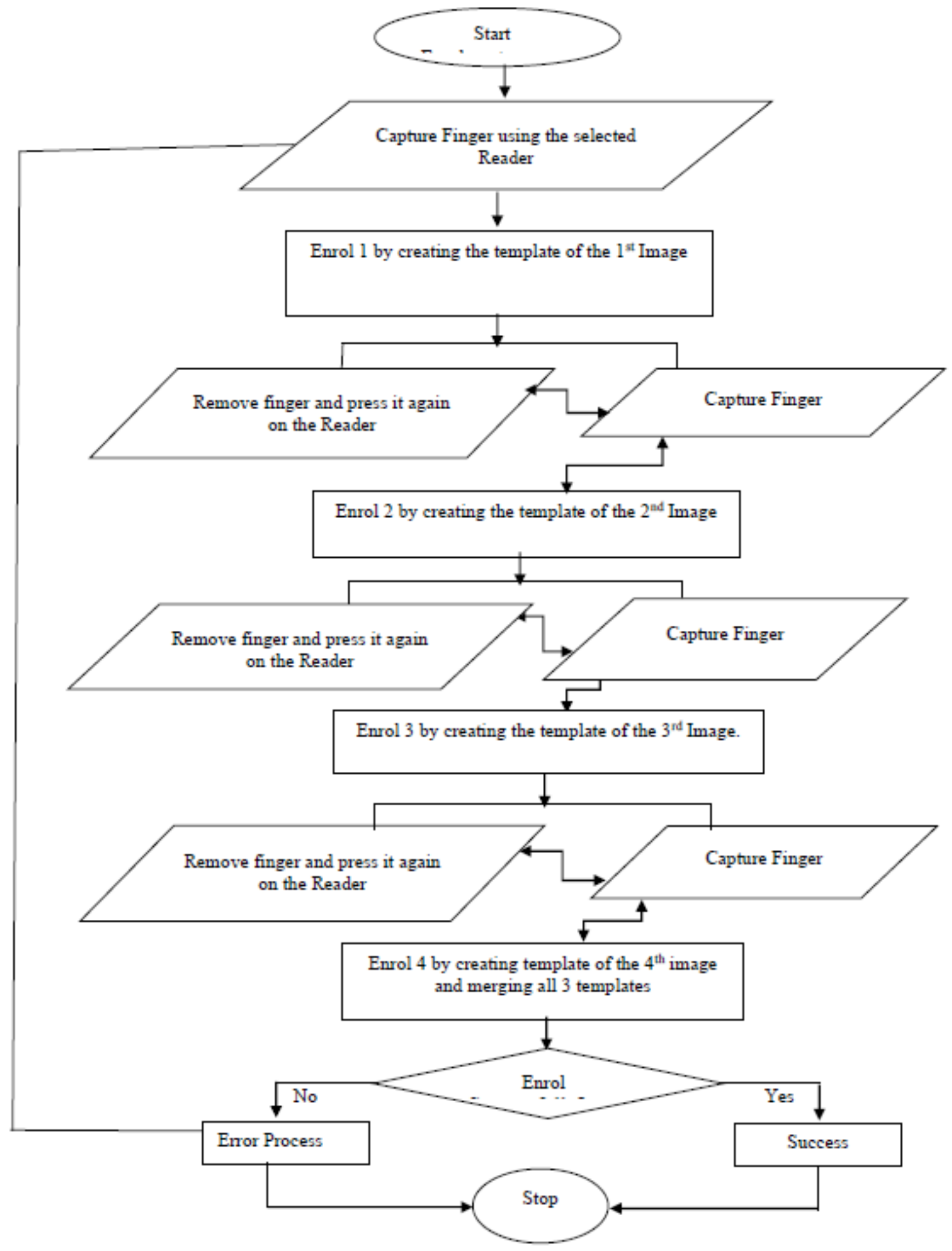

Figure 1: Enrolment Flowchart

(C) 2021 Faculty of Engineering, Lagos State University, Ojo. Nigeria. All rights reserved. 


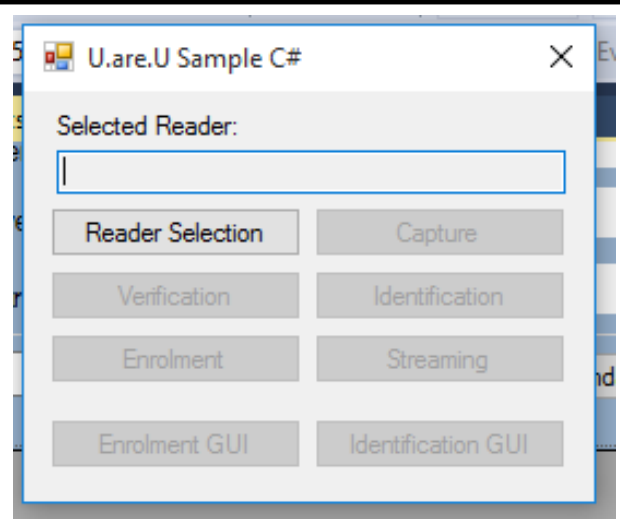

Figure 2: Main Menu of the System

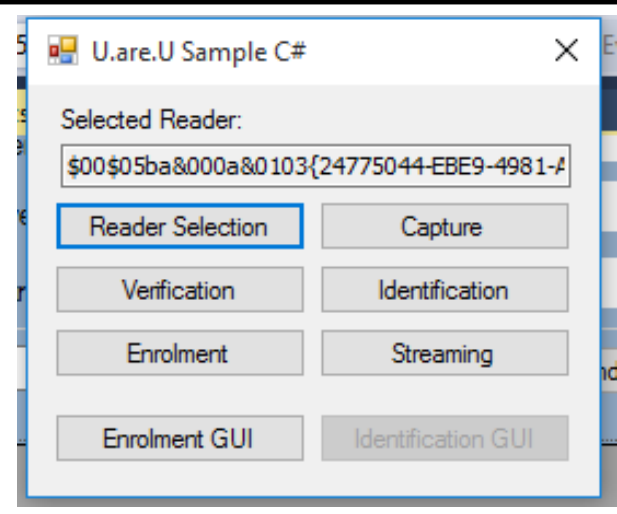

Figure 3: Reader Selection Interface

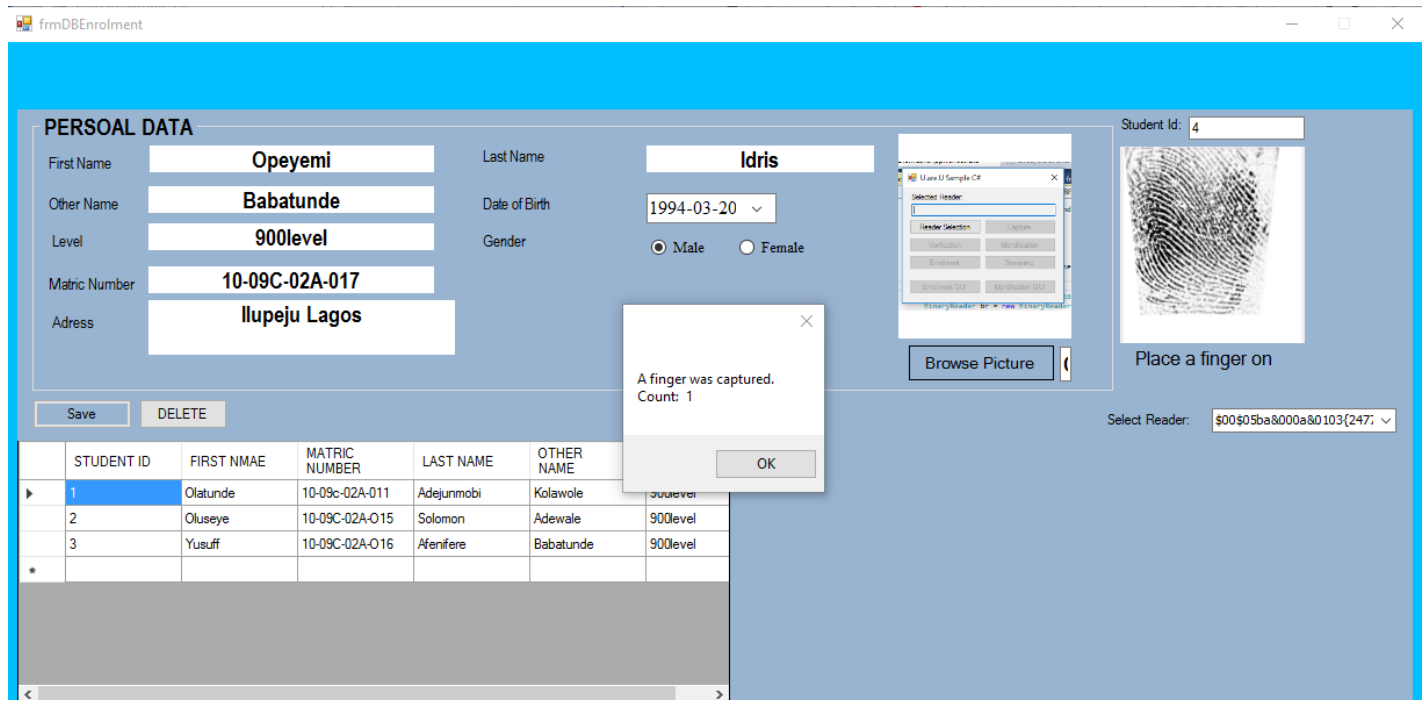

Figure 4: Enrolment Interface with Input I

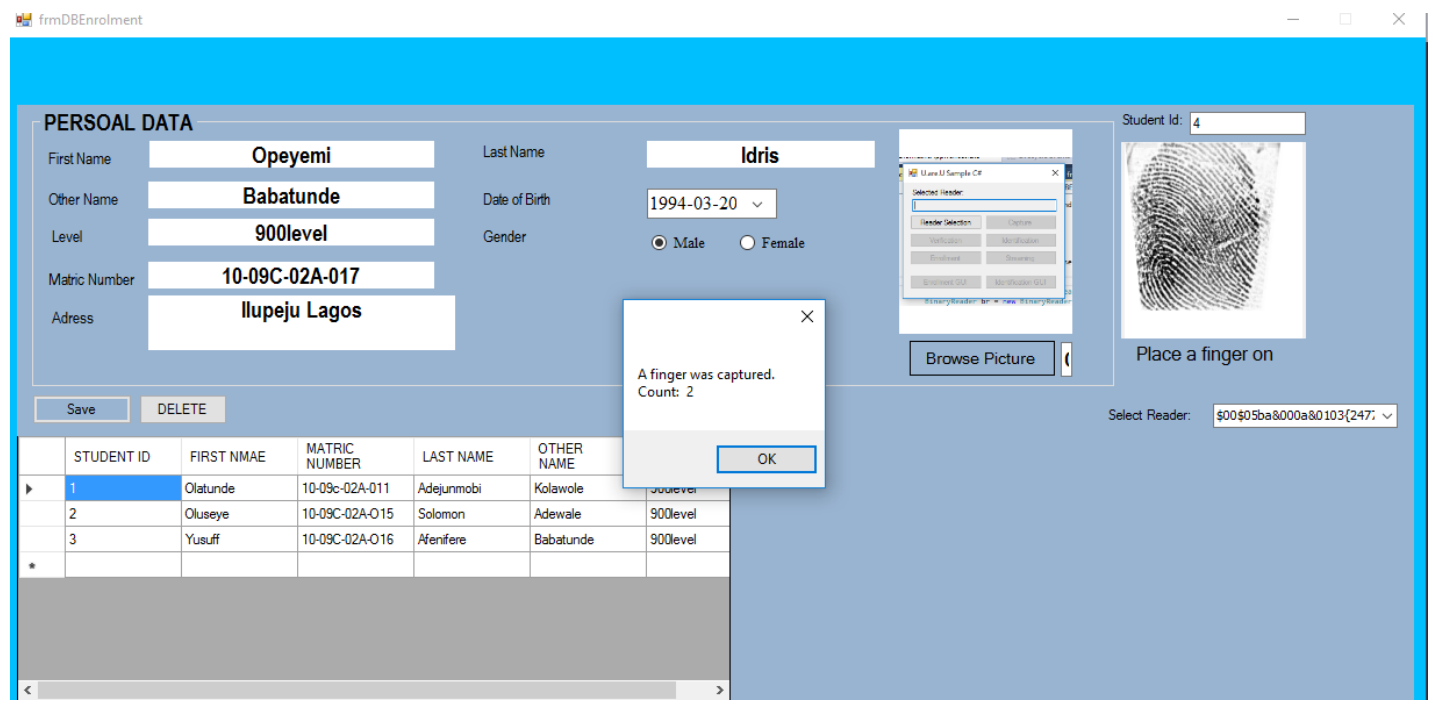

Figure 5: Enrolment Interface with Input II

(C) 2021 Faculty of Engineering, Lagos State University, Ojo. Nigeria. All rights reserved. 


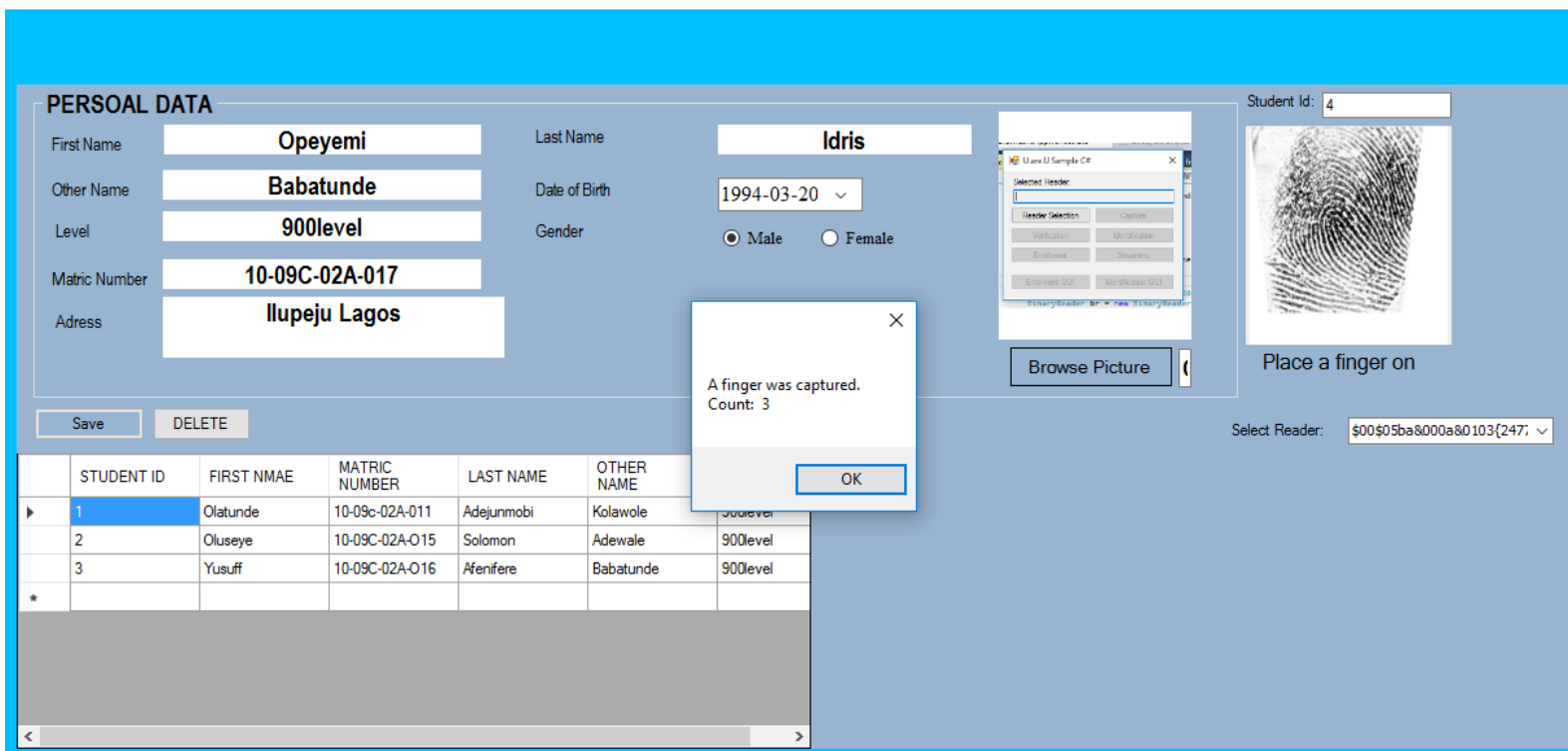

Figure 6: Enrolment Interface with Input III

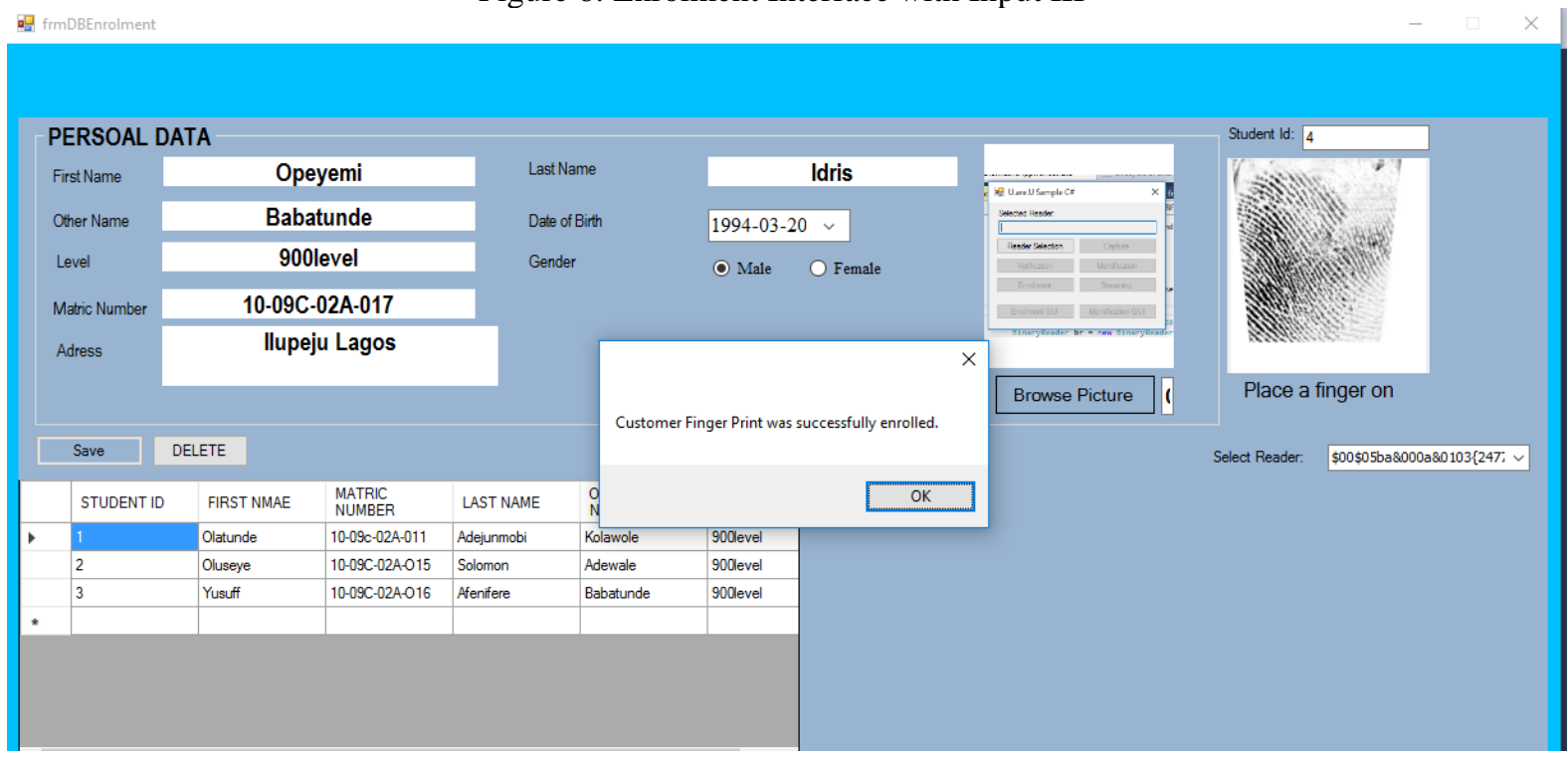

Figure 7: Enrolment Interface with Input IV

\section{RESULTS AND DISCUSSION}

In the model, Figure 2 shows the main menu that displays the different command buttons for each interface to work with. The first process is to select the type/name of Reader (fingerprint module) that will be used to enrol and verify students in the system's database. The other buttons have specific features and are mainly used for navigation:

Reader Selection Interface (Figure 2): This button is used to navigate to the interface where the user selects the type of fingerprint model to be used in the enrolment and verification processes.

Enrolment: This button is used to navigate to the enrolment interface where data entry and fingerprint enrolment processing takes place.

\section{Adejumobi et al, 2021}


Enrolment $\boldsymbol{G} U$ : This interface is used to check the effectiveness of fingerprint imagery and capturing.

Capture: Clicking this button will read and display finger lines and paths placed on the reader.

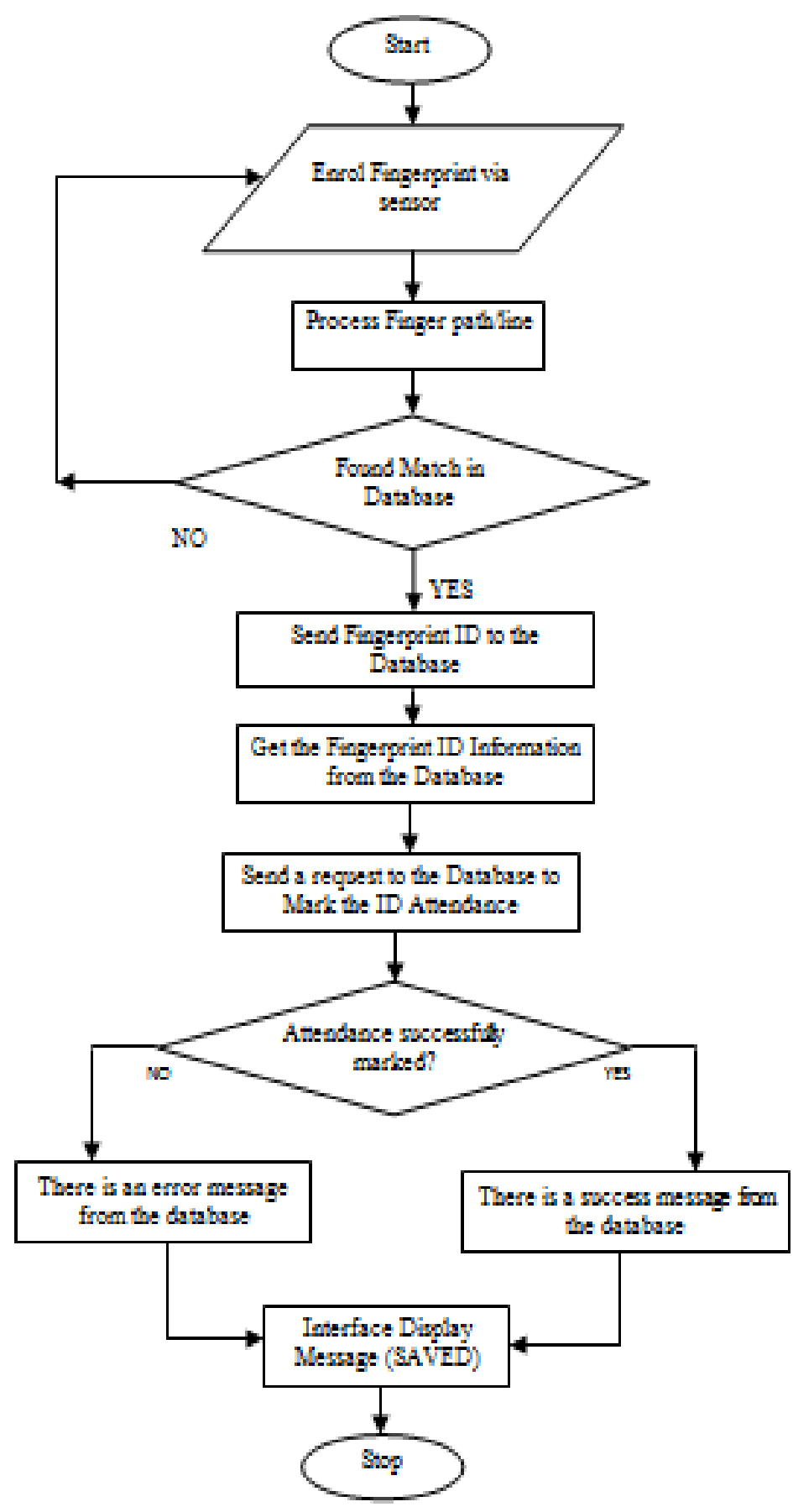

Figure 8. Fingerprint Recognition (Verification Flowchart) 
晿 frmDBVerify

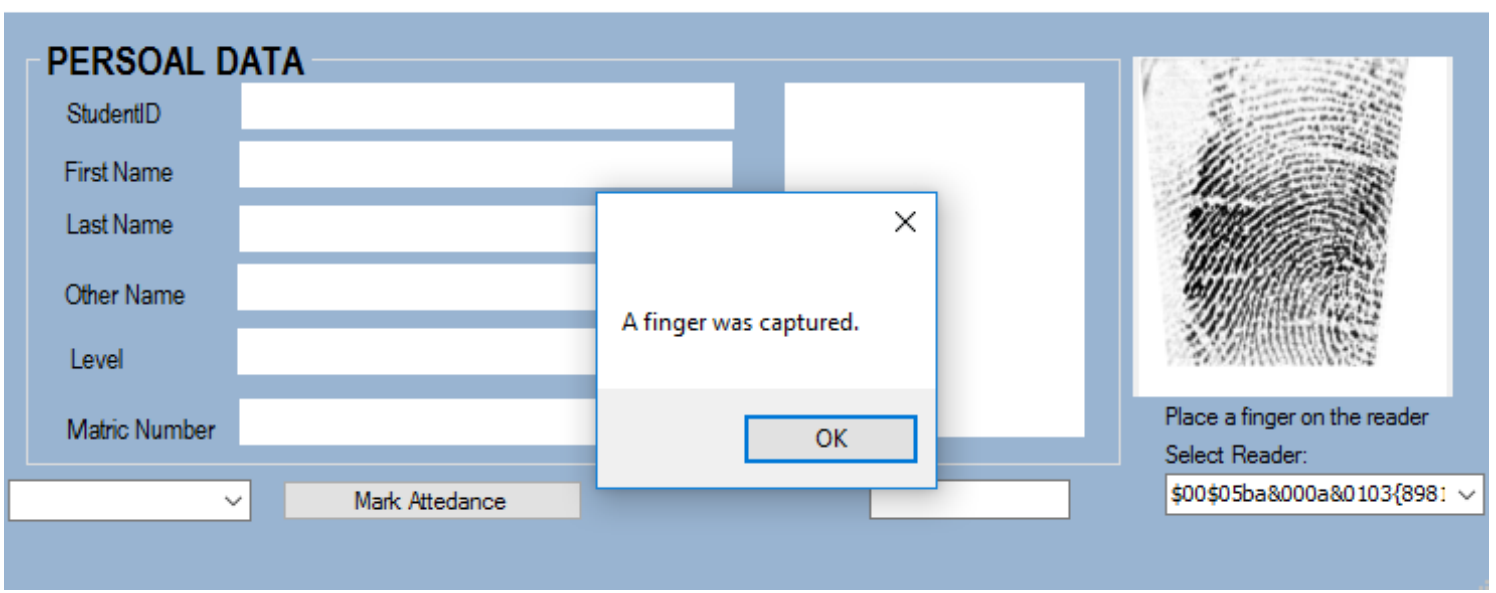

Figure 9. Verification Interface

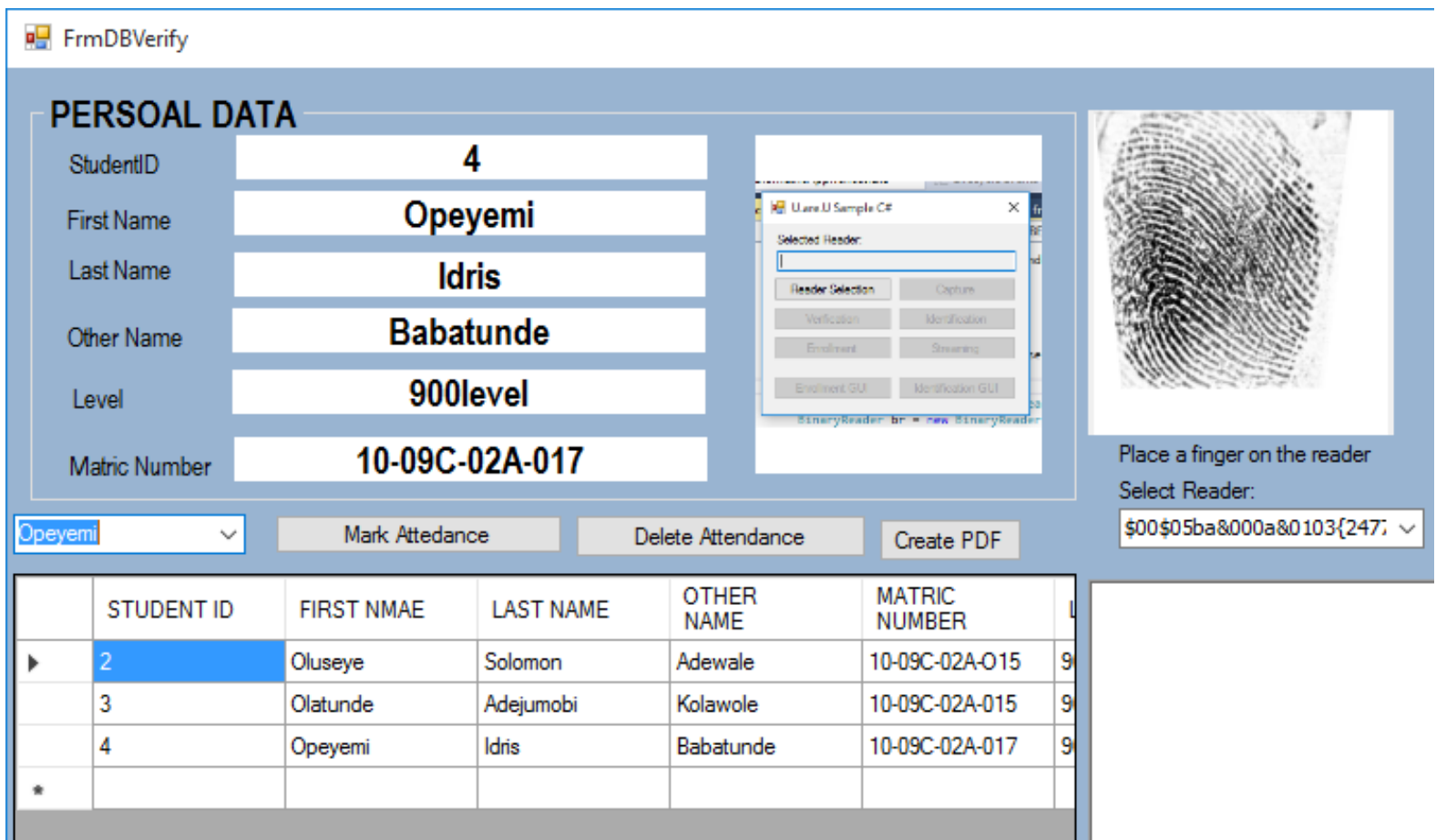

Figure 10: Verification Interface after Validation

\section{RESULTS DISCUSSION}

In the model, Figure 2 shows the main menu that displays the different command buttons for each interface to work with. The first process is to select the type/name of Reader (fingerprint module) that will be used to enrol and verify students in the system's database. The other buttons have specific features and are mainly used for navigation:

Reader Selection Interface (Figure 2): This button is used to navigate to the interface where the user selects the type of fingerprint model to be used in the enrolment and verification processes. 
Enrolment: This button is used to navigate to the enrolment interface where data entry and fingerprint enrolment processing takes place.

Enrolment $\boldsymbol{G} U$ : This interface is used to check the effectiveness of fingerprint imagery and capturing.

Capture: Clicking this button will read and display finger lines and paths placed on the reader.

Verification: This interface consists of four (4) important features namely; Mark Attendance, Create PDF, Student Fingerprint Verification Process, and the Mail Messaging Features. Once the enrolment of the students is completed and saved into the system's database, fingerprint recognition (or verification), and marking of the attendance are done during lecture periods. The verification process is done by placing a registered finger on the fingerprint reader; if found, the system will display the details of the student. The attendance is then marked and saved into the database with real date and time (see Figures 9 and 10). After marking the attendance of all students present for a particular lecture, the enrolment table in the system's database can be viewed as displayed in Figure 11. The fingerprints captured and the details of the students were converted into XML version code for verification processes. Figure 12 shows, the attendance of all students marked and recorded against real date and time.

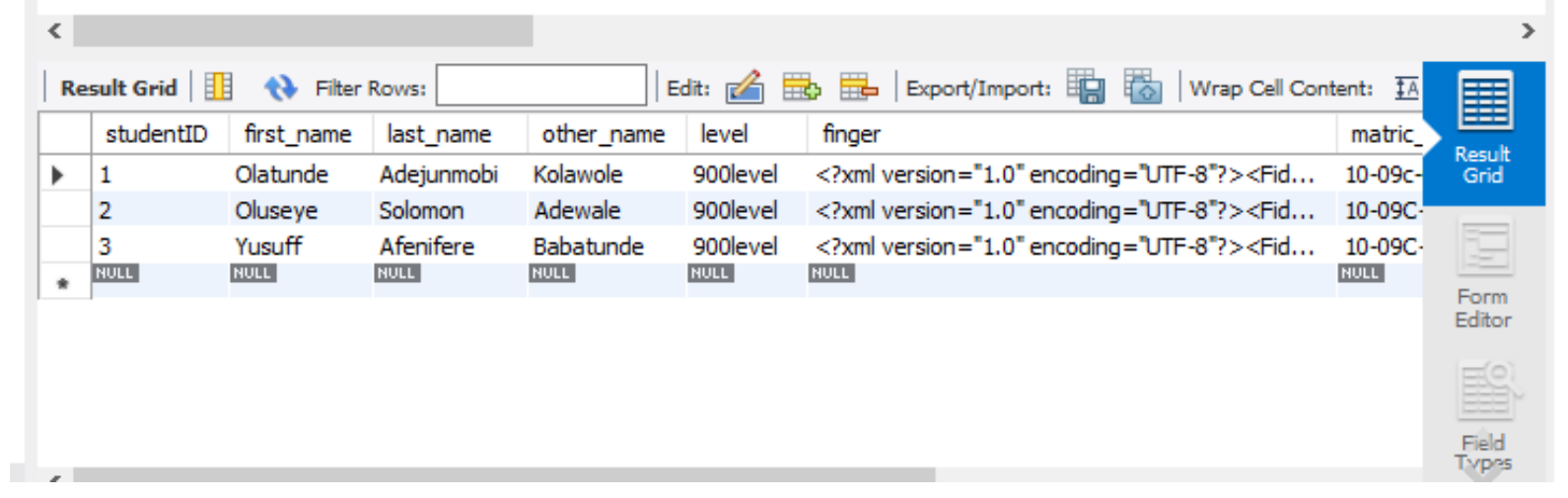

Figure 11: Enrolment Database Table

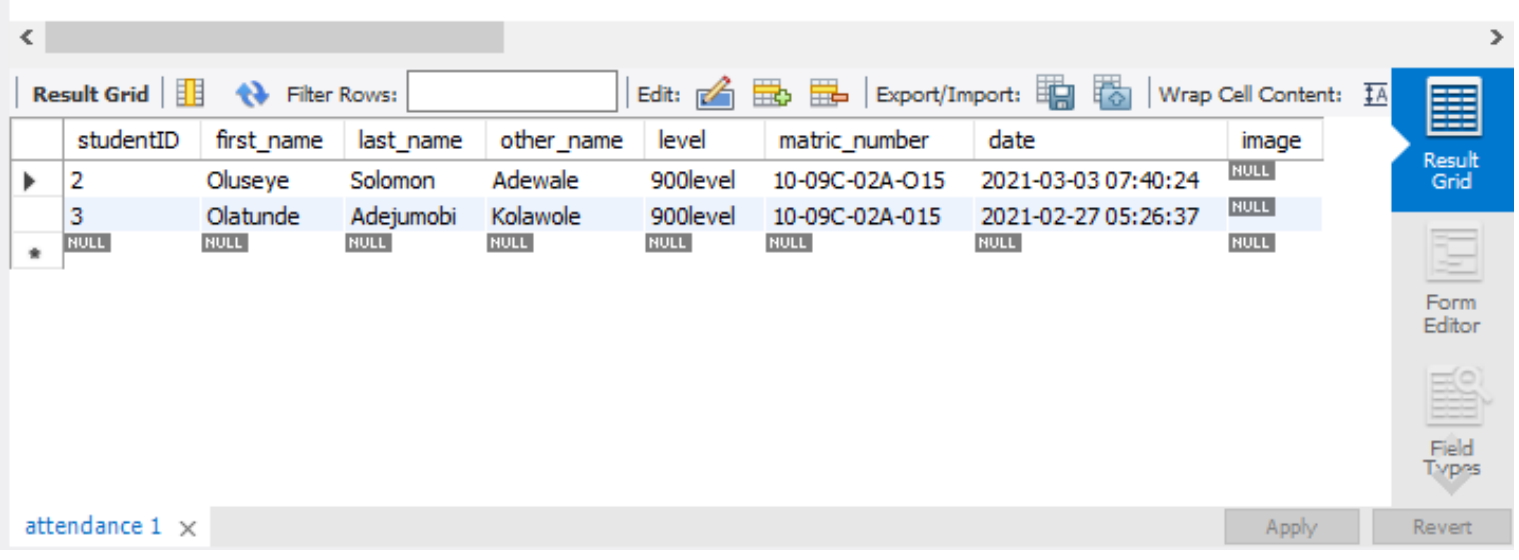

Figure 12: Attendance Database Table

The attendance for a specific lecture can be converted to PDF (see Figure 10). This is then sent to the e-mails of the head of department and the Parents via the SMTP feature (see Figure 13) to ascertain the levels of the students' attendance. 


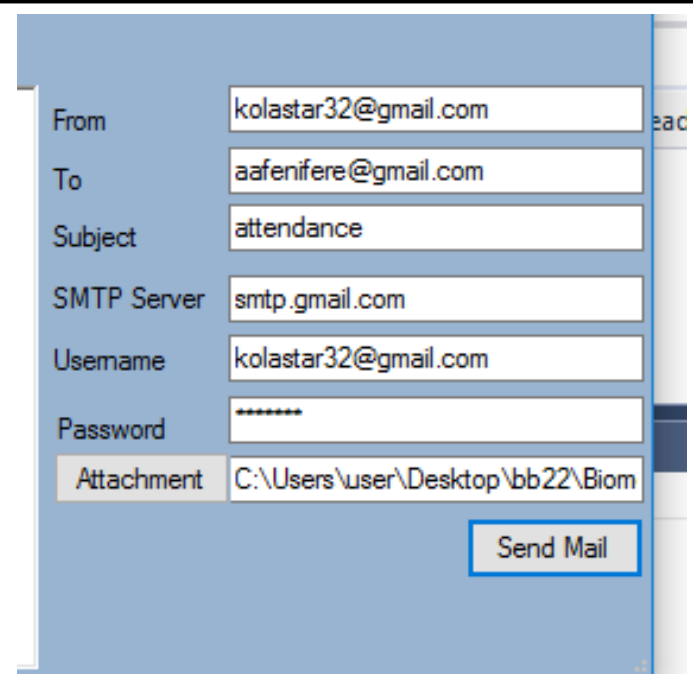

Figure 13: Mail Messaging Interface

\section{CONCLUSION}

The attendance system developed is fingerprint-based with Simple Message Transfer Protocol (SMTP). The enrolment and the verification interfaces are modelled using C\# while the database is MSQL based to make it more secure. The model is simple, not costly and a real-time system. The fingerprints of different students were successfully enrolled, stored in the database and later verified during the lecture period for attendance marking. The attendance record can be sent to relevant stakeholders to ascertain the levels of students' attendance,

\section{REFERENCES}

[1] Newman-Ford, L., Fitzgibbon, K., Lloyd, S., and Thomas, S. (2008) “A Large-Scale Investigation Into The Relationship Between Attendance And Attainment: A Study Using An Innovative Electronic Attendance Monitoring System". Studies in Higher Education, Vol. 33, No. 6. Pp. 699-717.

[2] Salami, I. O. (2020): “Biometric Automatic Attendance System in Nigeria Higher Institutions ". Global Scientific Journal (GSJ). Vol. 8, Issue 6. Pp 883 - 909.

[3] Kommey, B., Anyane-Lah O. and Amuzu, W. E. (2018): “SwyftTapp: An NFC Based Attendance System Using Fingerprint Authentication”. International Journal of Engineering, Science and Technology. Vol. 10, No. 1, pp. 23-39.

[4] Albahari, B., Drayton, P., \& Merrill, B. (2002). C\# Essentials: Programming the. NET Framework. " O'Reilly Media, Inc.".

[5] Setyawati, E., Wijoyo, H., \& Soeharmoko, N. (2020). Relational Database Management System (RDBMS).

[6] Shubham P. Uike, Monika P. Tambakhe, Chetna S. Dakhore, Waghade A. G. (2020): "Smart Student Attendance Management System”. International Journal of Innovations in Engineering and Technology (IJIET). Vol. 7, Issue 2. Pp 859 - 863.

[7] Rahman, S., Rahman, M., \& Rahman, M. M. (2018). Automated student attendance system using fingerprint recognition. Edelweiss applied science and technology, 2(1), 90-94.

[8] Karwan Jacksi, Falah Ibrahim, Shahab Ali (2018): “Student Attendance Management System”. Scholars Journal of Engineering and Technology (SJET). Vol. 6, Issue 2. Pp 49 - 53. 
[9] Devendra Kumar Yadav, Sumit Singh, Shashank Pujari, Pragyan Mishr (2015): "Fingerprint Based Attendance System Using Microcontroller and LabView". International Journal of Electrical, Electronics and Instrumentation Engineering. Vol. 4, Issue 6, Pp 5111- 5122.

[10] Hitesh Walia, Neelu Jain (2016): "Fingerprint Based Attendance Systems-A Review". International Research Journal of Engineering and Technology (IRJET). V01. 3, Issue 5. Pp 1166 - 1173.

[11] Srinidhi, M.D. and Roy, R. (2015): “A Web Enabled Secured System for Attendance Monitoring and Real Time Location Tracking Using Biometric and Radio Frequency Identification (RFID) Technology", IEEE International Conference on Computer Communication and Informatics (ICCCI), Coimbatore, India, Jan. 08- 10, 2015.

[12] Asdaque Hussain, M.D., Komirisetty Venkata Naga Krishna, Ramanulla Lakshmi Chandana, Velivela Krishna Chaitanya (2019): “An Efficient and Smart Attendance Management System". International Journal of Innovative Technology and Exploring Engineering (IJITEE). Vol. 8, Issue 7. Pp 2426 - 2430.

[13] Wang and Jingli (2015): "The Design of Teaching Management System in Universities Based on Biometrics Identification and the Internet of Things Technology". IEEE $10^{\text {th }}$ International Conference on Computer Science \& Education (ICCSE), Cambridge University, UK July 22-24, 2015, pp. 979-982.

[14] Potadar, M.P., Marathe, V.V., Khose, A.S. and Kotkar, L.A. (2015): "Biometric Attendance Recording and Communication System", International Journal of Innovations in Engineering and Technology (IJIET), Vol. 5, Issue 15. Pp. 230-234.

[15] Kamaraju, M. and Kumar, P.A. (2015): “Wireless Fingerprint Attendance Management System”, IEEE International Conference on Electrical Computer and Communication Technologies (ICECCT), March $5-7$.

[16] Shoewu, O., and Idowu, O.A. (2012): "Development of Attendance Management System using Biometrics", Pacific Journal of Science and Technology, Pp. 300-307. 